\title{
MODELING INTERNAL RADIATION THERAPY
}

\author{
Egon L. van den Broek \\ Human-Centered Computing Consultancy, Vienna, Austria \\ Human-Media Interaction (HMI), Faculty of EEMCS, University of Twente \\ P.O. Box 217, 7500 AE Enschede, The Netherlands \\ Karakter University Center, Radboud University Medical Center Nijmegen \\ P.O. Box 9101, 6500 HB Nijmegen, The Netherlands \\ vandenbroek@acm.org \\ http://www.human-centeredcomputing.com/ \\ Theo E. Schouten \\ Institute for Computing and Information Sciences, Radboud University Nijmegen \\ P.O. Box 9104, 6500 HE Nijmegen, The Netherlands \\ t.schouten@cs.ru.nl
}

Keywords: Radiation therapy, Modeling, Distance transform, FEED, Exact.

\begin{abstract}
A new technique is described to model (internal) radiation therapy. It is founded on morphological processing, in particular distance transforms. Its formal basis is presented as well as its implementation via the Fast Exact Euclidean Distance (FEED) transform. Its use for all variations of internal radiation therapy is described. In a benchmark trial, FEED proved to be truly exact as well as faster than a comparable technique. These features can be of crucial importance in radiation therapy as the balance between maximization of treatment effect and doses that cause unwanted damage to healthy tissue is fragile. This balance can be secured using the modeling technique presented here.
\end{abstract}

\section{INTRODUCTION}

We propose a new technique to model the treatment of malignant tumors through radiation therapy. This technique is founded on principles from morphological processing and computational geometry. It exploits the rather recent advances in imaging techniques that enable accurate modeling of malignant tumors (Ş. Iğdem et al., 2010; Zaidi et al., 2009). This technique facilitates both fast and exact modeling of radiation therapy by providing an accurate model of the malignant tumor.

As cancer is life threatening and clinicians are usually pressed for time, both speed and accuracy are of the utmost importance. Moreover, the effectiveness of the treatment heavily relies on the accuracy with which the radiation therapy can be employed. The fact that radiation therapy is usually given through fractions of radiation, instead of one dose, makes this even more challenging.

As this work is highly interdisciplinary, we will first provide a brief introduction on radiation therapy in Section 2 followed by a brief introduction on morphological processing in Section 3. This includes a small benchmark trial in which the proposed technique is compared with other related techniques. Next, in Section 4, the previous two sections will be linked and the proposed technique will be introduced. Lastly, in Section 5 we will present the conclusions and closing remarks.

\section{RADIATION THERAPY}

Radiation therapy (a.k.a. radiotherapy, x-ray therapy, and irradiation) is the application of ionizing radiation to eliminate cancer cells and shrink tumor tissue. The ionizing radiation damages and often destroys cells in the area being treated, as it damages the cells' genetic material. Approximately half of all cancer patients receive radiation therapy in the absence or presence of other treatments (e.g., chemotherapy or surgery).

Radiation therapy varies both in i) the types of radiation and ii) the method of its delivery. Consequently, radiation therapies can be executed with various characteristics. For example, a trade off can be made between the depth of penetration of and the control over the radiation. Not only the method of radiation therapy varies but also its goal. With the aim to 
cure the patient, the destruction of an entire tumor is the goal. With palliative radiation therapy, the aim is to provide relief for the patient; in this case the goal is usually to let the tumor shrink and spare healthy tissue as much as possible.

Radiation therapy is used to treat solid tumors, of almost every type (including leukemia and lymphoma). The radiation dose chosen depends on a number of factors including the type of cancer and the location of the tumor (i.e., whether or not it is near vulnerable tissue).

\subsection{Standards}

Three types of radiation therapy can be distinguished:

- external radiation therapy: from outside the body,

- internal radiation therapy (i.e., brachytherapy): placed inside the body, and

- systemic radiation therapy: throughout the body.

The radiation dose absorbed by the tissue is denoted in the unit cGy or Gy (i.e., respectively 1 and 100 rads, which was the previously used unit).

To maximize the destruction of cancer tissue and minimize the damage to healthy tissue, the total dose of radiation is usually given in a sequence of smaller doses (a.k.a. fractions), given within a certain time window.

In 1993, to facilitate the generation of a common language and models for the procedures at all radiotherapy centers the International Commission on Radiation Units and Measurements (ICRU) defined the goals for radiation therapy (Brianzoni et al., 2008). In a nutshell, these directives state that the type of radiation that needs to be given depends, amongst other things, on a) the type of cancer, b) the location, including the depth of the tumor in the body, c) the patient's general health and medical history, and d) other types of treatments possible. In practice, the ICRU emphasized that this implies that two main factors are central in making the choice of therapy (Brianzoni et al., 2008): the volume definition and the quantity of the radiation dose. To enable the best possible treatment, the oncologist employs PET imaging to obtain the best possible image (Ş. Iğdem et al., 2010).

\subsection{The Definition of Volume}

The definition of volume has multiple layers (Brianzoni et al., 2008), each having their own criteria; see also Figure 1:

- Gross Tumor Volume (GTV): the evident tumor.

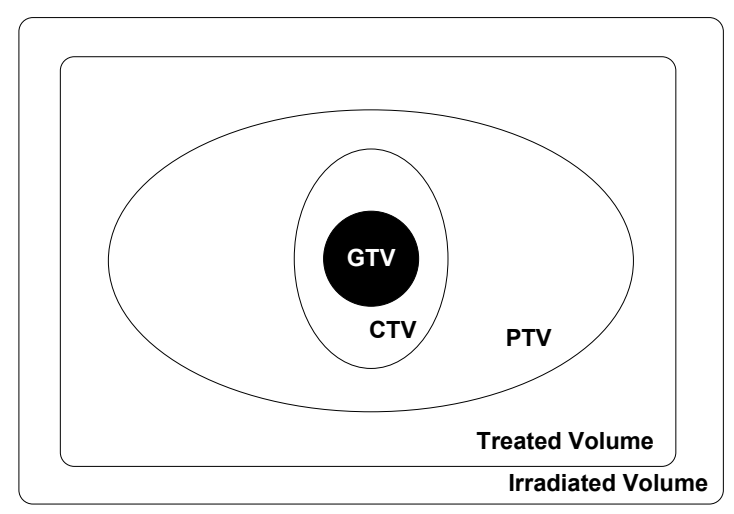

Figure 1: The volumes distinguished in planning radiation therapy, as defined by the International Commission on Radiation Units and Measurements (Brianzoni et al., 2008). GTV, CTV, and PTV denote respectively: gross tumor volume, clinical target volume, and planning target volume.

- Clinical Target Volume (CTV): the area surrounding the tumor that could have subclinical suspect disease. As such, the CTV is a clinical concept.

- Planning Target Volume (PTV): GTV, CTV, and a margin that takes into account a possible variation in shape, size, and position relative to the treatment beams and organ motion. The PTV, as a geometrical concept, which determines the prescribed dose in the CTV.

Ideally, the PTV is the same as the Treated Volume (TV) (see also Figure 1); however, in practice this is often not the case. In any case, the Irradiated Volume (IV) has to be taken into account. The IV receives radiation and its impact on normal tissue should be considered as well in radiation therapy treatments. Self evidently, the match between PTV and TV is of the utmost importance.

In general, it is assumed that the dose of radiation is near to homogeneous inside the PTV. Nevertheless, it is important that radiation therapy is always evaluated. In particular, the level of homogeneity registered near the reference point doses and both the minimum and maximum value inside the PTV have to be evaluated. Several imaging techniques are employed for evaluation purpose: computed tomography (CT), ultrasound, or magnetic resonance imaging (MRI). For optimal results, these are often combined with positron emission tomography (PET) (Ş. Iğdem et al., 2010). The latter imaging technique is complementary to the former three.

In the next section, we will switch from the clinical part of this study to its technical part. We start by defining the basics on morphological processing. Subsequently, we describe distance transformations, the one applied in particular, and a benchmark trial. 


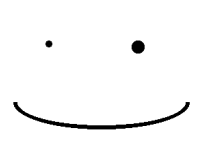

(a)

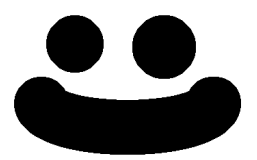

(b)

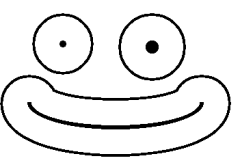

(c)

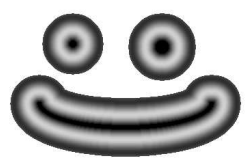

(d)

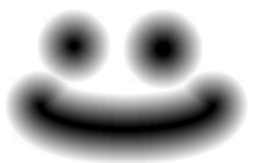

(e)

Figure 2: Five representations of the same test image, which illustrates the width of distance transforms (DT) that can be applied: (a) the original, (b) after dilation, (c) DT that provides the extremes, (d) a discrete DT, and (e) a gradual DT.

\section{MORPHOLOGICAL PROCESSING}

The image elements needed to represent malignant tumor tissue can be extracted using morphological image processing. Morphological operators can be conveniently described using set theoretic notation. Their implementation is less straightforward as is illustrated by the plethora of algorithms introduced (Borgefors et al., 2003; Fabbri et al., 2008; Razmjooei and Dudek, 2010).

Dilation (a.k.a. dilatation) and erosion are fundamental morphological image processing operations. Many of the morphological algorithms applied are founded on these two primitive operations.

Given two sets $A$ and $B$ in $\mathbb{Z}^{2}$, the dilation of $A$ by $B$, is defined as

$$
A \oplus B=\left\{x \mid(B)_{x} \cap A \neq \emptyset\right\},
$$

where $(B)_{x}$ denotes the translation of $B$ by $x=$ $\left(x_{1}, x_{2}\right)$, which is defined as:

$$
(B)_{x}=\{c \mid c=b+x, \exists b \in B\}
$$

Hence, $A \oplus B$ expands $A$ if the origin is contained in $B$, as is often the case.

The erosion of $A$ by $B$, denoted $A \ominus B$, is the set of all $x$ such that $B$ translated by $x$ is completely contained in $A$, which is defined as

$$
A \ominus B=\left\{x \mid(B)_{x} \subseteq A\right\}
$$

Hence, $A \ominus B$ decreases $A$.

Founded on these two morphological operations, the 4- $n$ and the 8- $n$ dilation algorithms were developed (Rosenfeld and Pfaltz, 1966) for region growing purposes. These region growing algorithms are founded on the city-block distance and the chessboard distance measure. For $4-n$ and $8-n$ growth for an isolated pixel at the origin, the set of pixels contained in the dilated shape are respectively defined as:

$$
\begin{gathered}
C_{4}(n)=\left\{(x, y) \in \mathbb{Z}^{2}:|x|+|y| \leq n\right\}, \\
C_{8}(n)=\left\{(x, y) \in \mathbb{Z}^{2}:|x| \leq n,|y| \leq n\right\},
\end{gathered}
$$

where $n$ is the number of iterations.

To obtain a better approximation for the Euclidean distance (ED), Rosenfeld and Pfaltz recommended the alternate use of the city-block and chessboard motions as early as 1966 . This defines the octagonal distance, which provides a better approximation of the ED than either of the distances separately.

\subsection{Distance Transformation (DT)}

Region growing algorithms can be applied to obtain a distance transformation (DT). A DT (Rosenfeld and Pfaltz, 1966) creates an image in which the value of each pixel is its distance to the set of object pixels $O$ in the original image:

$$
D(p)=\min \{\operatorname{dist}(p, q), q \in O\}
$$

The Euclidean DT (EDT) has been extensively used in image processing and pattern recognition, either by itself or as an important intermediate or ancillary method in applications. Examples of medical imaging methods that often involve EDT are: the analysis of functional MRI data (Lu et al., 2003), image registration (Salvi et al., 2007), and image segmentation (Mazonakis et al., 2001).

Several methods for the calculation of EDT have been introduced over the years, both for sequential and parallel machines (Fabbri et al., 2008; Razmjooei and Dudek, 2010; Schouten and van den Broek, 2010). However, in most cases this did not involve exact EDT, but only approximations; for example, (Cuisenaire and Macq, 1999; Razmjooei and Dudek, 2010).

Unlike existing approaches such as (Cuisenaire and Macq, 1999), we implemented EDT starting directly from Equation 6, or rather its inverse: each object pixel $o$, in the set of object pixels $(O)$ feeds its ED to all background pixels $b$ :

$$
\mathrm{D}(b)=\text { if }(b \in O) \text { then } 0 \text { else } \infty .
$$

Subsequently, the adapted naive algorithm is: 
Table 1: Timing results and the errors for three image sets on the city-block (or Chamfer 1,1) transform (Rosenfeld and Pfaltz, 1966), 2-scan method (EDT-2) (Shih and Wu, 2004), and the Fast Exact Euclidean Distance (FEED) (Schouten and van den Broek, 2004; Schouten and van den Broek, 2010), which is the only one that truly provides an exact EDT.

\begin{tabular}{lrrrrrr}
\hline \multicolumn{2}{l}{ Images } & \multicolumn{2}{c}{ Timing (in sec.) } & \multicolumn{2}{c}{ Algorithms } & \multicolumn{2}{c}{ Errors (in \%) } \\
& City-block & EDT-2 & FEED & City-block & EDT-2 & FEED \\
\hline standard objects & 8.75 & 38.91 & 17.14 & 2.39 & 0.16 & - \\
rotated objects & 8.77 & 38.86 & 18.02 & 4.66 & 0.21 & - \\
larger objects & 8.64 & 37.94 & 19.94 & 4.14 & 0.51 & - \\
\hline average & 8.72 & 38.57 & 18.37 & 3.73 & 0.29 & - \\
\hline
\end{tabular}

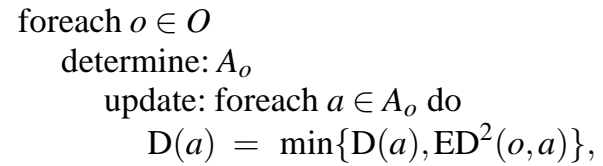

where $A_{o}$ is the area where $o$ should feeds distances to. This results in an exact but computationally inexpensive algorithm for EDT, which was baptized: Fast Exact Euclidean Distance (FEED) transformation (Schouten and van den Broek, 2004).

\subsection{Benchmark}

A small benchmark trial was executed in which FEED was compared with two other algorithms:

- 2-scan method (EDT-2) (Shih and Wu, 2004), which should be preferred over (Cuisenaire and Macq, 1999) fast EDT. This is the fastest EDT algorithm and, as such, the ultimate test for FEED.

- The city-block (or Chamfer 1,1) distance (Rosenfeld and Pfaltz, 1966), which served as a baseline.

Table 1 presents both the timing results and the errors for the three algorithms as obtained through the benchmark. With a rough estimation of the ED, the city block distance outperformed the other two algorithms by far with respect to execution times. Surprisingly FEED was $>2 \times$ as fast as EDT-2. However, more important than the algorithms' execution times are the errors they made compared to the exact EDT.

The city-block transform resulted for all three image types in an error-level of less than 5\%; see Table 1. (Shih and Wu, 2004) claimed that their two scan algorithm (EDT-2) provides exact EDs. However, although their algorithm was precise with $99 \%$ precision, EDT-2 was not exact; see also Table 1. So, FEED appeared to be the only algorithm that provided truly exact ED for all instances. As such, FEED is of interest to modeling radiation therapy, where speed is important but exactness is even more important as it determines the success of the therapy.

\section{MODELING}

In the previous two sections, the two ingredients of this paper were introduced. In this section we will bring both together and show how DTs, in particular FEED, are of interest to radiation therapy. With this, we may be introducing a new class of algorithms for modeling radiation therapy.

Radiation therapy is among the main treatments for cancer. As stated in Section 2 there are three types of radiation therapy. We will show that morphological processing can be of use for internal radiation therapy as well as for external radiation therapy. However, we will discuss the former type but will not discuss details concerning the latter one. Morphological processing is of no use for systemic radiation therapy.

So far, the DTs and their resulting distance maps have been introduced as a basic, generic technique. This is indeed the case; however, as stated in Section 3.1, DTs know many applications (Borgefors et al., 2003; Fabbri et al., 2008). We pose that radiation therapy could and should become one of them.

Instead of test images (see Figure 2) or abstract objects (see Table 1), we will take an image of a malignant tumor as object. Nowadays imaging techniques provide excellent means to localize tumor tissue, as was mentioned in Section 2. So, let us assume that a malignant tumor $T$ has been successfully identified on an image. Then, all other space on the image is denoted as background $b$; cf. Eqs. 7 and 8 .

Let us now denote both the tumor $T$ and the already defined background $b$ as two types of background: $b_{T} b_{s}$. Subsequently, the radiation doses $o$ $(\in O$, with $O$ denoting the complete radiation therapy) can be placed within $b_{T}$. Elements $o$ transmit their radiation that can be described via functions that serve as distance measures. In this way, all elements of the DT are described in terms of radiation therapy.

Having all elements defined, FEED can be applied and a distance map can be generated; see also Section 3.1. This distance map provides a map of the 


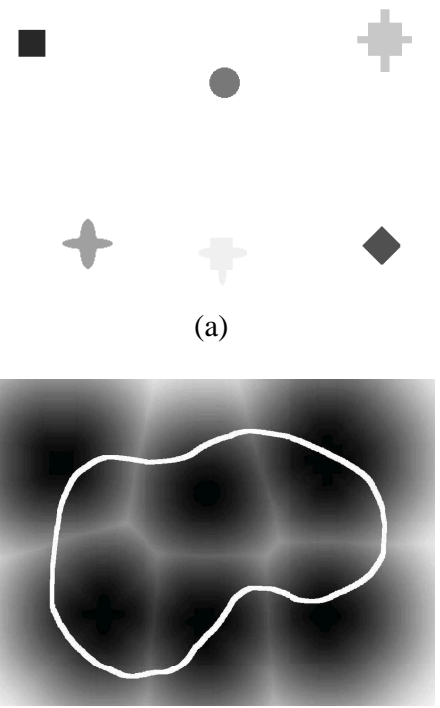

(b)

Figure 3: A model of radiation treatment of the central part of the planning target volume (PTV; see also Section 2.2 and Figure 1): (a) Six radiation doses, indicated by arbitrary shapes. (b) A distance map as generated by FEED, which models the amount of radiation delivered over the area. Intensity indicates the amount of radiation. The white line indicates the border of the malignant tumor.

radiation in both $b_{T}$ (i.e., the malignant tumor) and $b_{s}$ (i.e., the tissue surrounding the malignant tumor); see also Section 3. The radiation dose each cell receives is calculated in this way, which can be conveniently presented graphically. Figure 3a presents an artificially created volume and six radiation doses placed within it. The distribution of the radiation is visualized in Figure $3 b$, where the intensity denotes the amount of radiation, in this case as determined according to an arbitrary function.

Radiation therapy involves more than the binary distinction malignant tumor and healthy tissue, it employs volumes, as described in Section 2.2. After the radiation oncologist has defined the GTV, CTV, PTV, $\mathrm{TV}$, and IR, the radiation in these distinct volumes can be easily determined as well. So, for each volume the quantity of the radiation dose can be easily modeled in advance, which is one of the two main issues in radiation therapy; see also Section 2.1.

To maximize the destruction of the malignant tumor and minimize the damage to healthy tissue, the total dose radiation $O$ is usually given in a sequence of smaller doses $o$, given within a certain time window (Brianzoni et al., 2008). So, a sequence of DTs has to be conducted over time to model this. In its most simple form, this process can be modeled by a linear combination of the distance maps to each other (Censor et al., 2008).

As denoted in Section 2.1, other treatments may accompany radiation therapy. One of the possibilities is that multiple radiation therapies are conducted, each having their own characteristics (Brianzoni et al., 2008). Such a process can be modeled similarly as the sequence of radiation doses.

When applying multiple radiation therapies, it can be of interest to know which therapy has its biggest impact on which parts of the patient. Each therapy then has its distance map or output matrix. Then, the radiation therapy that provides the maximum radiation can be placed in a second output matrix. The maximum radiation dose indicates the amount of certainty (or weight) that the pixel belongs to the class. In this way, a matrix is generated with the therapy labels as elements. This can also be visualized by different color ranges for each therapy. In addition, a multi-therapy distance map can be generated.

\section{DISCUSSION}

This paper introduced the application of distance transformations (DT) for modeling radiation therapy. It started with brief introductions on radiation therapy, which illustrated the importance of the accuracy of treatment, and morphological processes (e.g., DT). Subsequently, we described how DT can be applied to model radiation therapy. We described how the different volumes as denoted in radiation therapy can be taken into account, how sequences of radiation doses can be modeled and even how multiple simultaneously conducted radiation therapies can be modeled.

This paper introduced a vivid approach for modeling radiation therapy. However, the technique proposed awaits thorough testing. Two types of tests can be distinguished: a) the application of the technique on data gathered from radiation oncologists and b) a benchmark of the technique proposed with established techniques currently employed. Moreover, this modeling technique did not yet take into account a range of complicating factors such as the complex functions that describe the transfer of radiation through several types of tissue. So, it is evident that a vast amount of work has to be done before the technique introduced here can establish itself.

This article assumed 2D images of the malignant tissue. However, 3D radiation therapy has already been introduced. This is particularly useful for external radiation therapy, where radiation beams go through the patient's body. Such 3D images can be obtained using CT, MRI, PET, or single photon emission computed tomography (SPECT) (Ş. Iğdem et al., 2010). Although not discussed in this article, FEED can also be applied on 3D images (Schouten et al., 
2006) and video (Schouten and van den Broek, 2010).

A relatively new type of therapy is IntensityModulated Radiation Therapy (IMRT) (Bortfeld, 2006). IMRT utilizes radiation beams of varying intensities to transmit different doses of radiation to small areas of tissue simultaneously. This provides the means to transmit higher doses of radiation within the malignant tumor and lower doses to healthy tissue (cf. Figure 1). As discussed in the previous section, sequences of radiation doses are no problem to model. This is no different when these doses have different intensities and is independent of whether or not they are given in sequence or in parallel.

On the whole, this article introduced a new technique to model internal radiation therapy. On the one hand, its use still has to be shown on medical data sets and it has to be compared with other established techniques. On the other hand, i) its formal basis and implementation are sound; ii) its use for all variations of internal radiation therapy is described as well; and iii) it has been shown to be exact as well as fast. This $100 \%$ accuracy, in particular, can be of crucial importance for radiation therapy purposes as the balance between maximization of treatment effect and doses that cause unwanted damage to healthy tissue is fragile (Censor et al., 2008). The future will learn whether or not this technique can indeed secure this balance and, consequently, will redeem its promises.

\section{ACKNOWLEDGEMENTS}

We thank the three anonymous reviewers for their constructive suggestions. Further, we thank Lynn Packwood for proof reading this article.

\section{REFERENCES}

Borgefors, G., Nyström, I., and Sanniti di Baja, G. (2003). Discrete geometry for computer imagery. Discrete Applied Mathematics, 125(1):[special issue].

Bortfeld, T. (2006). IMRT: A review and preview. Physics in Medicine and Biology, 51(13):R363-R379.

Brianzoni, E., Rossi, G., and Proietti, A. (2008). Gross Tumor Volume and Clinical Target Volume: Anatomical Computed Tomography and Functional FDG-PET, volume 2, chapter 13, pages 225-234. Burlington, MA, USA: Elsevier Academic Press.

Censor, Y., Galvin, J. M., Xiao, Y., and Langer, M. (2008). Linear and nonlinear models and algorithms in intensity-modulated radiation therapy (IMRT). Linear Algebra and its Applications, 428(5-6):12031206.
S. Iğdem, Alço, G., Ercan, T., Ünalan, B., Kara, B., Geceer, G., Akman, C., Zengin, F. O., Atilla, S., and Okkan, S. (2010). The application of Positron Emission Tomography/Computed Tomography in radiation treatment planning: Effect on gross target volume definition and treatment management. Clinical Oncology, 22(3):173-178.

Cuisenaire, O. and Macq, B. (1999). Fast Euclidean transformation by propagation using multiple neighborhoods. Computer Vision and Image Understanding, 76(2):163-172.

Fabbri, R., da F. Costa, L., Torelli, J. C., and Bruno, O. M. (2008). 2D Euclidean distance transform algorithms: A comparative survey. ACM Computing Surveys, 40(1):Article 2.

Lu, Y., Jiang, T., and Zang, Y. (2003). Region growing method for the analysis of functional MRI data. $\mathrm{Neu}$ roImage, 20(1):455-465.

Mazonakis, M., Damilakis, J., Varveris, H., Prassopoulos, P., and Gourtsoyiannis, N. (2001). Image segmentation in treatment planning for prostate cancer using the region growing technique. British Journal of Radiology, 74(879):243-249.

Razmjooei, S. and Dudek, P. (2010). Approximating Euclidean distance transform with simple operations in cellular processor arrays. In IEEE Proceedings of the 12th International Workshop on Cellular Nanoscale Networks and their Applications (CNNA), pages 181185, Berkely, CA, USA. IEEE.

Rosenfeld, A. and Pfaltz, J. L. (1966). Sequential operations in digital picture processing. Journal of the ACM, 13(4):471-494.

Salvi, J., Matabosch, C., Fofi, D., and Forest, J. (2007). A review of recent range image registration methods with accuracy evaluation. Image and Vision Computing, 25(5):578-596.

Schouten, T. E., Kuppens, H. C., and van den Broek, E. L. (2006). Three dimensional fast exact Euclidean distance (3D-FEED) maps. Proceedings of SPIE (Vision Geometry XIV), 6066:60660F.

Schouten, T. E. and van den Broek, E. L. (2004). Fast Exact Euclidean Distance (FEED) Transformation. In Kittler, J., Petrou, M., and Nixon, M., editors, Proceedings of the 17th IEEE International Conference on Pattern Recognition (ICPR 2004), volume 3, pages 594-597, Cambridge, United Kingdom.

Schouten, T. E. and van den Broek, E. L. (2010). Incremental Distance Transform (IDT). In Erçil, A., Çetin, M., Boyer, K., and Lee, S.-W., editors, Proceedings of the 20th IEEE International Conference on Pattern Recognition (ICPR), pages 237-240, Istanbul, Turkey.

Shih, F. Y. and Wu, Y.-T. (2004). Fast Euclidean distance transformation in two scans using a $3 \times 3$ neighborhood. Computer Vision and Image Understanding, 93(2):195-205.

Zaidi, H., Vees, H., and Wissmeyer, M. (2009). Molecular $\mathrm{PET} / \mathrm{CT}$ imaging-guided radiation therapy treatment planning. Academic Radiology, 16(9):1108-1133. 